\title{
Effect of ticagrelor on acute kidney injury in septic rats and its underlying mechanism
}

\author{
CHENG YU*, CUI-MIN GAO*, NA XIE, XIAO-QING WANG and YU-QING MA \\ Department of Anesthesiology, First Hospital of Lanzhou University, Lanzhou, Gansu 730000, P.R. China
}

Received September 28, 2019; Accepted December 11, 2020

DOI: $10.3892 / \mathrm{etm} .2021 .9906$

\begin{abstract}
The aim of the present study was to determine whether the effects and underlying mechanisms of ticagrelor in a rat model of sepsis-induced acute kidney injury (AKI) were mediated via the CD62P pathway. A total of 15 rats were randomly assigned to the following groups: Normal, sham, cecal ligation and puncture (CLP), CLP + clinical dose of ticagrelor (CCD) and CLP + loading dose of ticagrelor (CLD). Ticagrelor was administered $12 \mathrm{~h}$ before modeling, immediately after modeling, and $12 \mathrm{~h}$ after modeling at a dose of 8.6 and $46.42 \mathrm{mg} / \mathrm{kg}$ in the CCD and CLD groups, respectively. Rats in the normal, sham and CLP groups were treated with the same volume of distilled water. Serum creatinine ( $\mathrm{SCr}$ ), $\mathrm{CD} 62 \mathrm{P}$ and interleukin-1 $\beta$ (IL-1 $\beta$ ) levels, myeloperoxidase (MPO) activity in the renal tissue and the apoptosis rate of renal cells were increased in the CLP group, compared with in the normal and sham groups $(\mathrm{P}<0.05)$. In addition, ticagrelor treatment reduced SCr, CD62P and IL-1 $\beta$ expression levels, renal tissue MPO activity and renal cell apoptosis in rats with sepsis-induced AKI $(\mathrm{P}<0.05)$. CD62P expression was closely associated with the occurrence of sepsis-induced AKI. The mechanism of ticagrelor-mediated reductions in inflammation, renal neutrophil infiltration and renal cell apoptosis is possibly associated with reductions in CD62P expression.
\end{abstract}

\section{Introduction}

Sepsis is one of the most common causes of acute kidney injury (AKI) in critically ill patients (1). Compared with AKI caused by other causes, sepsis-induced AKI increases the length of hospital stay, medical resource consumption and mortality, and adversely affects the overall clinical prognosis of patients (2).

Correspondence to: Professor Yu-Qing Ma, Department of Anesthesiology, First Hospital of Lanzhou University, 1 West Donggang Road, Lanzhou, Gansu 730000, P.R. China

E-mail:myq2392466@163.com

${ }^{*}$ Contributed equally

Key words: sepsis, acute kidney injury, ticagrelor, inflammation, apoptosis, CD62P
The mortality rate of toxic patients after kidney injury is as high as $70 \%$ (3), whilst the survival rate of renal replacement therapy is $13.8 \%$ (4). Previous studies have reported that the pathophysiological mechanism of septic AKI may be associated with overactivation of the immune system, uncontrolled inflammation and dysfunction of the coagulation system and energy metabolism $(5,6)$.

The kidney has two sets of microcirculatory structures, namely the glomerulus and peritubular microcirculatory networks located in the renal cortex and renal medulla (7). The dense distribution and complex structure of the capillary network of the kidney make it more susceptible to oxidative stress and inflammation, rendering the kidney highly susceptible to hypoperfusion and hypoxia (7). Adaptive changes occur in an environment with unbalanced oxygen consumption, including apoptosis of tubular epithelial cells (TECs) or even necrosis (8). In addition, abnormal activation of white blood cells and coagulation disorders are also causes of AKI (9). The aforementioned mechanisms make the kidney one of the organs most susceptible to sepsis.

A previous study has demonstrated that the severity of sepsis is associated with platelet activation (10). Platelets are widely involved in the processes of coagulation, inflammatory factor release and immune cell activation (9). Platelet activation dependent granule external membrane protein (PADGEM or CD62P, also known as P-selectin) protein is expressed on the surface of the $\alpha$-granule membrane secreted after platelet activation and is a marker of platelet activation (11). It serves a key role in the process of platelet-mediated immune cell activation (12). Ticagrelor is a novel anti-platelet drug with a strong effect and is primarily used for the prevention and treatment of acute coronary syndrome in clinical practice by targeting the P2Y12 receptor (13). It can inhibit platelet activation and reduce the secretion of CD62P (14). In the present study, the aim was to explore the mechanism of platelet activation of CD62P protein in septic AKI, and the effect of ticagrelor in decreasing the secretion of CD62 in a septic AKI rat model, in an attempt to provide theoretical support for the prevention or treatment of septic AKI.

\section{Materials and methods}

Animals and study design. Included in the present study were 50 healthy male 8-week-old SD rats (weighing 260-300 g; Chinese Academy of Agricultural Sciences of Institute of 
Veterinary Medicine) after adaptation to the standard laboratory conditions with free access to food and water except modeling. The temperature in the vivarium was $21-22^{\circ} \mathrm{C}$, the humidity was $40-60 \%$ and the day/night cycle was $13 / 11 \mathrm{~h}$. The animals were equally randomized into five groups: i) Normal group (without laparotomy and intervention); ii) sham group (laparotomy without intervention); iii) group receiving cecal ligation and puncture without any treatment (CLP); iv) group receiving a clinical dose of $8.6 \mathrm{mg} / \mathrm{kg}$ body weight ticagrelor (CCD); and v) group receiving a loading dose of $46.42 \mathrm{mg} / \mathrm{kg}$ body weight of ticagrelor (CLD). Ticagrelor was administered via oral gavage $12 \mathrm{~h}$ before modeling, immediately after modeling and $12 \mathrm{~h}$ after modeling; the different time points were based on the pharmacokinetics of ticagrelor according to the drug instructions (15). Ticagrelor was provided by the Department of Medicinal Chemistry, AstraZeneca Branch, Lanzhou, China. The present study was approved by the Research Ethics Committee of the First Hospital of Lanzhou University (Lanzhou, China).

CLP critical procedure. After routine shaving and disinfection of the abdominal area, a mid-line incision $(\sim 10 \mathrm{~mm})$ was made after successful induction of anesthesia via intraperitoneal (i.p.) injection of $1 \%$ pentobarbital sodium $(40 \mathrm{mg} / \mathrm{kg})$. The cecum in the lower left area of the abdominal cavity was exposed. Two transmural injuries were made to the ligated section following cecal ligation using polyglactin sutures using a 20-gauge needle to allow peritoneal dissemination of bacteria. The abdominal cavity was then closed by applying corresponding sutures, followed by fluid resuscitation. Animals in the sham group underwent the same procedure without cecal puncture and ligation, and animals in the normal group did not undergo any procedures.

Preparation and detection of serum and platelet-rich plasma $(P R P)$. After $24 \mathrm{~h}$ modeling, $1 \%$ pentobarbital sodium $(40 \mathrm{mg} / \mathrm{kg})$ was injected i.p. into the abdomen. Whole blood drawn from the heart was centrifuged at $4^{\circ} \mathrm{C}$ and $1,700 \mathrm{x} \mathrm{g}$ for $15 \mathrm{~min}$ to obtain serum samples. Serum was stored at $-20^{\circ} \mathrm{C}$, and serum creatinine (SCr) and interleukin- $1 \beta$ (IL-1 $\beta$ ) levels were measured using ELISAs (SCr, cat. no. ml059158; https://www.mlbio.cn/goods-59158.html; IL-1 $\beta$ cat. no. ml037361; https://www.mlbio.cn/goods-37361. html; Shanghai Enzyme-linked Biotechnology Co., Ltd.). The remaining blood was injected into the anticoagulant tube and centrifuged at 2,700 $\mathrm{x}$ g for $5 \mathrm{~min}$ to isolate PRP at $4^{\circ} \mathrm{C}$, and the level of CD62P was detected using an ELISA (cat. no. ml028431; Shanghai Enzyme-linked Biotechnology Co., Ltd.; https://www.mlbio.cn/goods-28431.html). After the blood was collected, the rats were still under anesthesia and were euthanized using $25 \%$ potassium chloride $(75 \mathrm{mg} / \mathrm{kg})$ until the heart stopped.

Kidney biopsy process and histological lesions. After $24 \mathrm{~h}$ modeling and whole blood removal, rats were euthanized using $25 \%$ potassium chloride $(75 \mathrm{mg} / \mathrm{kg})$ until the heart stopped before the bilateral kidneys were removed. The right kidney was immediately stored in a $-80^{\circ} \mathrm{C}$ refrigerator, and the left kidney was fixed using $10 \%$ formaldehyde in room temperature $\left(20-25^{\circ} \mathrm{C}\right)$ and $>8 \mathrm{~h}$, embedded in paraffin, sliced into $4-\mu \mathrm{m}$-thick sections and stained using hematoxylin and eosin (H\&E) $5 \mathrm{~min}$ in room temperature. All the tissue sections were independently evaluated in a blinded manner under an optical microscope by the investigators (magnification, x400; cat. no. BX51-32H01; Olympus Corporation). The scores were determined as follows: i) 0 , not detected, such that the glomeruli and renal tubules were unchanged; ii) 1 , where $1-10 \%$ tubules involved, part of the renal tubules were occluded, the brush border of the renal tubular cells was absent; iii) 2, 10-25\% tubules involved; iv) 3, 25-50\% tubules involved; and v) $4,>50 \%$ of tubules involved (16).

Determination of renal tissue homogenate. The right kidney tissue was prepared into a $10 \%$ tissue homogenate with pre-cooled saline and renal cortex, and centrifuged at $4{ }^{\circ} \mathrm{C}$ and $1,300 \mathrm{x} g$ for $20 \mathrm{~min}$ to obtain the supernatant. To determine the neutrophil content in the renal tissue, an ELISA (cat. no. ml003250; Shanghai Enzyme-linked Biotechnology Co., Ltd.; https://www.mlbio.cn/goods-3250.html) was used to detect myeloperoxidase (MPO) activity, as the MPO level represents an indicator of global tissue neutrophil recruitment (17).

Assessment of renal cell apoptosis. This procedure was performed as previously described (18). Fresh kidney tissue fragments were added to a sufficient volume of type II collagenase digestive solution (cat. no. 17101-015; Thermo Fisher Scientific, Inc.) and digested in a thermostatic shaker at $37^{\circ} \mathrm{C}$ for $5 \mathrm{~min}$. The protected sample was incubated for $15 \mathrm{~min}$ at $37^{\circ} \mathrm{C}$ away from light and gently agitated every $5 \mathrm{~min}$. All supernatants were collected and centrifuged at $4{ }^{\circ} \mathrm{C}$ and $70 \mathrm{x}$ g for $5 \mathrm{~min}$. The bottom sediment was removed by filtration through a 200-mesh stainless steel mesh to remove the residual tissue and then centrifuged at $30 \mathrm{xg}$ for $2 \mathrm{~min}$ at $4^{\circ} \mathrm{C}$. The supernatant was discarded, and the isolated kidney cells were collected and counted under a fluorescence microscope (magnification, $x 400$ ). The cells were stained and protected from light using an FITC-Annexin V Apoptosis Detection kit at room temperature $\left(20-25^{\circ} \mathrm{C}\right.$, stain for $15 \mathrm{~min}$ ) stored at $4^{\circ} \mathrm{C}$ (cat. no. C1062S; Beyotime Institute of Biotechnology) and renal cell apoptosis was detected using the BD FACSVerse ${ }^{\mathrm{TM}}$ flow cytometer (BD Biosciences) analyzed by Flowjo (V10.5.2; BD Biosciences) and inverted fluorescence microscopy (magnification, x400; cat. no. IX53-DP73; Olympus Corporation). There was not enough renal tissue to repeat the staining protocol whilst including a stain such as DAPI in the fluorescence experiments, which represented a limitation of the present study.

Statistical analysis. Statistical analysis was performed using the SPSS 16.0 statistical software package (SPSS, Inc.). The measurement data are expressed as the mean $\pm \mathrm{SD}$. One-way ANOVA and Tukey's post hoc test were used for comparison between groups. Non-parametrically distributed data was analyzed using Kruskal-Wallis with Dunn's post hoc test. $\mathrm{P}<0.05$ was considered to indicate a statistically significant difference.

\section{Results}

General condition of rats. No significant change was noticed in the rats of the normal group. The rats in the sham group were 
all in a good mental condition after surgery, with normal water feeding, stable breathing and sensitive to stimuli. Meanwhile, septicemia symptoms, such as refusal to eat and drink, drowsiness, hair erection and diarrhea were observed in CLP group, and hematuria appeared in some rats. Compared with the CLP group, the aforementioned conditions were milder in CCD and CLD groups, but more severe than in the sham group.

Histological findings. The effect of ticagrelor on sepsis-induced renal histological alterations was evaluated using $\mathrm{H} \& \mathrm{E}$ staining (16). The results indicated that the glomeruli and renal tubules were unchanged in the normal group, where cells were arranged regularly, the structure was intact, the nucleus was central, the nucleolus was clear and the plasma was evenly stained. The findings in sham group were similar to those in the normal group, except that the tubular cells were slightly edematous. In the CLP group, interstitial congestion, edema and inflammatory cell infiltration were observed in the renal tissue, and part of the renal tubules were occluded, the brush border of the renal tubular cells was absent, and the top surface of the cell membrane was vacuolated and protruded into the lumen. Parts of the renal tubules were compensated for dilatation, the epithelium of the tube was flat, the nucleus disappeared, the basement membrane was exposed and the Tamm-Horsfall protein cast was observable. In the CCD group, the structure of the renal tubules was less damaged, with no luminal occlusion, less inflammatory cell infiltration, slight damage to the renal tubular epithelial cells and edema of vacuolated changes. In the CLD group, only tubular cell edema was seen, and no inflammatory cell infiltration was observed. Compared with the sham group, the injury score were increased in the CLP, CCD and CLD groups $(\mathrm{P}<0.05)$. Moreover, compared with the CLP group, the injury score were decreased in the CCD and CLD groups $(\mathrm{P}<0.05$; Fig. 1$)$.

Changes in SCr, CD62P, IL-1 $\beta$ and renal tissue MPO levels. Renal function is usually represented by $\mathrm{SCr}$ levels, platelet activity by CD62P levels, the degree of neutrophil accumulation in the kidney by MPO activity, and inflammatory factor levels by IL-1 $\beta$ levels (19-22). Compared with the normal and sham groups, SCr, CD62P, IL-1 $\beta$ and renal tissue MPO were increased in the CLP group $(\mathrm{P}<0.05)$. Moreover, compared with the CLP group, SCr, CD62P, IL-1 $\beta$ and renal tissue MPO were decreased in the CCD and CLD groups $(\mathrm{P}<0.05)$. Finally, compared with the CCD group, CD62P in the PRP of the CLD group was decreased ( $\mathrm{P}<0.05$; Fig. 2).

\section{Cell apoptosis in the kidney}

Flow cytometric analysis of the renal cell apoptosis rate. In the normal and sham groups, some early apoptotic cells of Annexin $\mathrm{V}^{+}$/propidium iodide (PI)- were observed, but very few Annexin $\mathrm{V}^{+} / \mathrm{PI}^{+}$intermediate and advanced apoptotic cells or secondary necrotic cells were observed. Compared with the normal and sham groups, a large number of Annexin $\mathrm{V}^{+} / \mathrm{PI}^{-}$early apoptotic cells and Annexin $\mathrm{V}^{+} / \mathrm{PI}^{+}$intermediate-to-advanced apoptotic cells or secondary necrotic cells were observed in the CLP group, with significant differences between the groups $(\mathrm{P}<0.05)$. Compared with the CLP group, the number of early, intermediate and advanced apoptotic cells was significantly decreased in the CCD and CLD groups $(\mathrm{P}<0.05)$. The change in renal cell apoptosis was consistent with that of $\mathrm{SCr}$ in all groups, suggesting that renal cell apoptosis was associated with septic AKI (Fig. 3).

Inverted fluorescence microscopy of renal cell apoptosis. Inverted fluorescence microscopy for double-stained cells revealed that: i) The early apoptotic cell membrane showed green fluorescence (FITC fluorescence); ii) the intermediate and advanced stage apoptotic cell nucleus exhibited red fluorescence (PI fluorescence) and the cell membrane displayed green fluorescence; and iii) only red fluorescent nuclei were seen in late apoptotic and dead cells, and the cell membranes were disintegrated. Few green fluorescent cell membranes were observed in the normal and sham groups. Large numbers of cells were observed with red-fluorescent nuclei and green-fluorescent membranes or with red-fluorescent nuclei only in the CLP group, while in the CCD and CLD groups there were primarily green fluorescence-stained cells, and some nuclei were stained with red fluorescence. In addition, there were more cells that stained red in the CCD group compared with CLD groups (Fig. 4).

\section{Discussion}

Ticagrelor is widely used for the prevention and treatment of acute coronary syndrome in clinical practice. It is the first reversible $\mathrm{P} 2 \mathrm{Y} 12$ receptor antagonist that can effectively decrease platelet activation and aggregation (8). Ticagrelor inhibits further release of the platelet agonist adiponectin from dense particles by antagonizing the P2Y12 receptor, thereby achieving the effect of inhibiting platelet activation and aggregation (23). Compared with clopidogrel, ticagrelor has a more potent antiplatelet effect (14). Moreover, pharmacokinetics reveal that the inhibitory effect of ticagrelor on platelets is concentration-dependent, there is a longer time window between antithrombotic and bleeding accidents, and its clinical safety is higher (23). A previous study have demonstrated that ticagrelor can also mimic the anti-inflammatory effect of adenosine (24). However, there are few studies reporting the use of ticagrelor for the treatment of septic AKI. The aim of the present study was to explore the association between CD62P and septic AKI, and the effect of ticagrelor on septic AKI.

Sepsis is a serious medical condition with complex pathogenesis, resulting in high morbidity and mortality (25). The exact molecular mechanisms underlying this disease are yet to be elucidated and effective therapeutic targets need to be developed. In the present study, a rat model of sepsis-induced AKI was established via CLP. Postoperatively, the rats were generally presented in a poor condition. Pus was observed in the abdominal cavity of the sacrificed animals and the ligated cecal segments became black and necrotic. SCr level was increased and the pathological sections revealed that the kidney tissue was severely damaged. All these findings indicated that the rat septic AKI model was constructed successfully.

A clinical study has previously demonstrated that the severity of sepsis was associated with platelet activation (16). During the activation of platelets after sepsis, CD62P is converted from the internal storage protein of the cell to a platelet membrane surface protein, eventually being expressed 


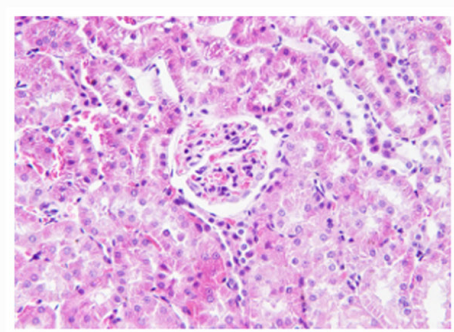

Normal

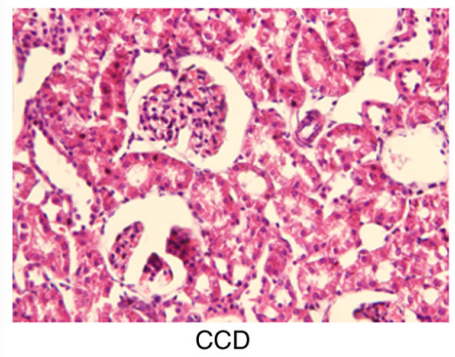

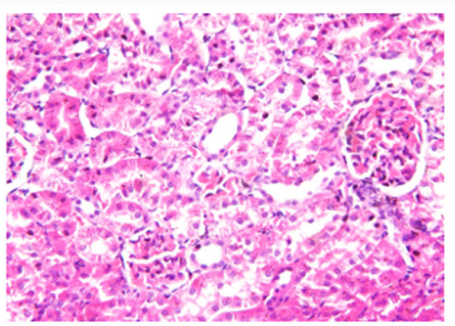

Sham

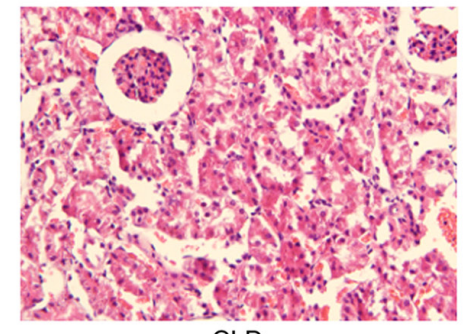

CLD

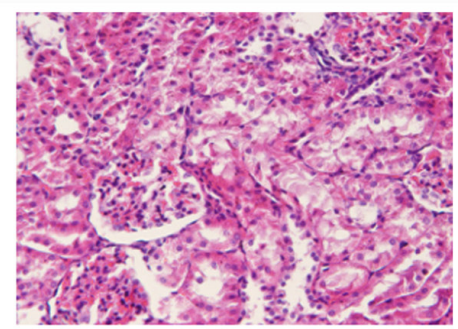

CLP

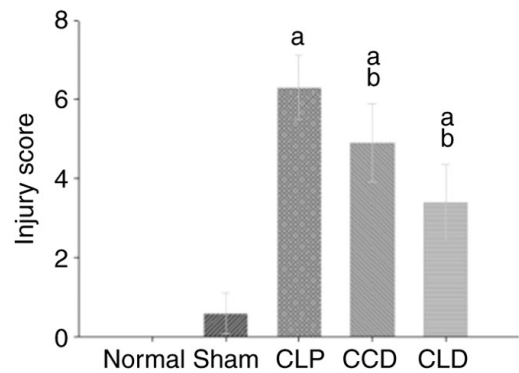

Figure 1. Histological features of the renal tissues in different groups as examined by H\&E staining. Microscopic observation of histological properties in renal tissues obtained from different groups of mice (magnification, $\mathrm{x} 400 ; 250 \pm 25$ cells $/ 0.245 \mathrm{~mm}^{2}$ ); $\mathrm{n}=10 / \mathrm{group}$. ${ }^{\mathrm{a}} \mathrm{P}<0.05 \mathrm{vs}$. sham; ${ }^{\text {b }} \mathrm{P}<0.05 \mathrm{vs}$. CLP. CLP, cecal ligation puncture; CCD, CLP + clinical dose of ticagrelor; CLD, CLP + loading dose of ticagrelor.
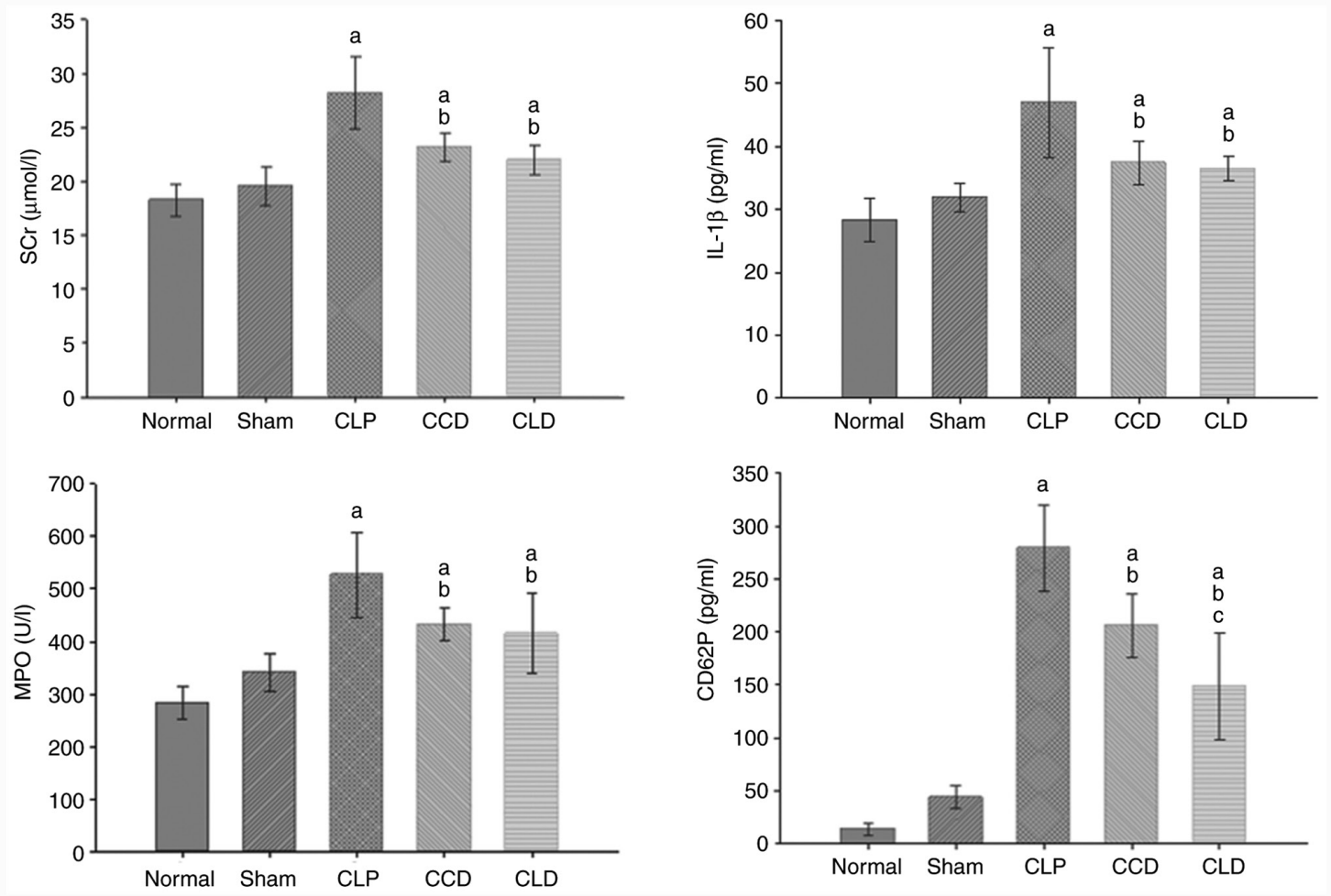

Figure 2. Effects of ticagrelor on SCr, IL-1 $\beta$, MPO and CD62P levels in rats. N=10/group. ${ }^{\text {a }}<0.05$ vs. sham; ${ }^{\text {P }}<0.05$ vs. CLP; ${ }^{c} \mathrm{P}<0.05$ vs. CCD. CLP, cecal ligation puncture; CLP + clinical dose of ticagrelor; CLD, CLP + loading dose of ticagrelor; IL, interleukin; MPO, myeloperoxidase; SCr, serum creatinine.

on the surface of platelet cell membranes as cell adhesion receptors; thus, it represents a marker for detecting platelet activation (26). The present study demonstrated that the CD62P concentration in septic AKI animals of the CLP group was higher than that in normal and sham groups, indicating that CD62P was to some extent associated with septic AKI.

Lerolle et al (27) found extensive infiltration of neutrophils in the glomeruli, renal interstitia and capillaries upon autopsy of patients with sepsis AKI. The authors therefore speculated that accumulation of neutrophils in the kidney was involved in the pathogenesis of septic AKI. In the present study, in the CLP group, H\&E staining of the renal tissue revealed that inflammatory cells accumulated in the kidney, renal tubular epithelial cells were damaged and Tamm-Horsfall protein casts were observed, indicating that the occurrence of septic AKI was partly associated with the accumulation of renal inflammatory cells. 

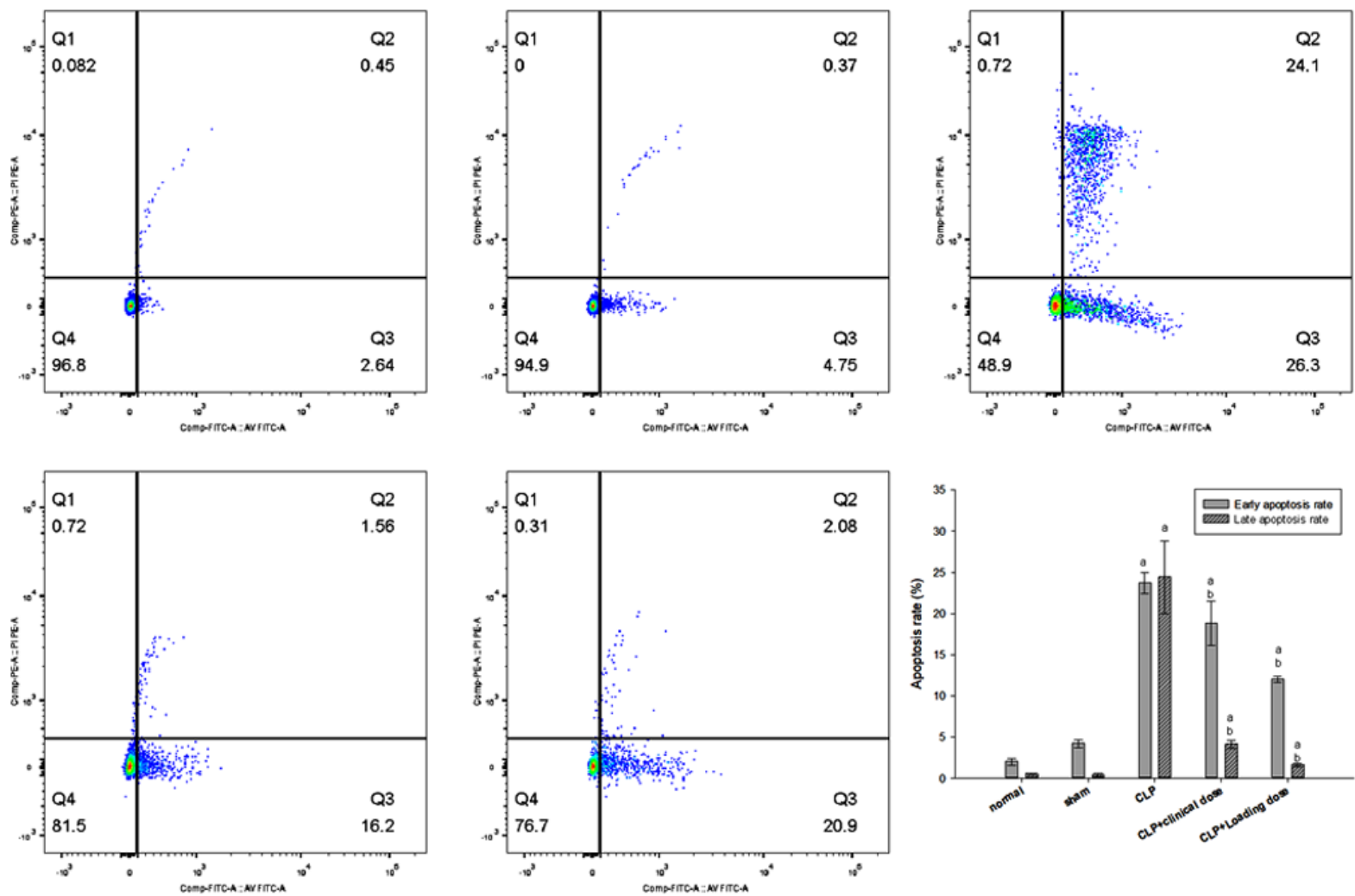

Figure 3. Renal cell apoptosis in rats. Flow cytometry analysis of isolated kidney cells, which was quantified; $\mathrm{n}=5 / \mathrm{group}$. ${ }^{\mathrm{a}} \mathrm{P}<0.05$ vs. sham and normal; ${ }^{\mathrm{b}} \mathrm{P}<0.05$ vs. CLP. CLP, cecal ligation puncture; CCD, CLP + clinical dose of ticagrelor; CLD, CLP + loading dose of ticagrelor; FITC-A, FITC-Annexin V; PI, propidium iodide.

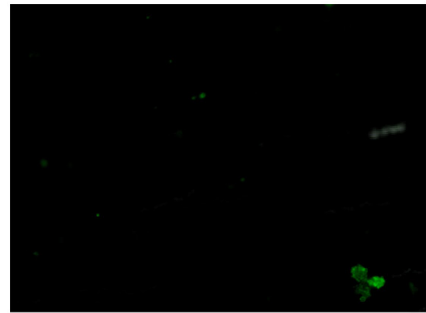

Normal

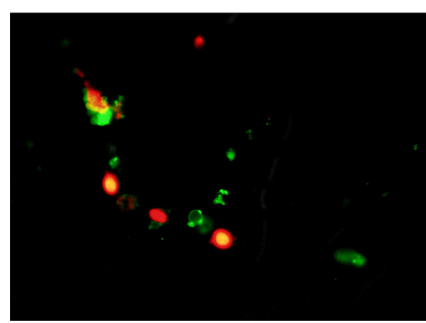

CCD

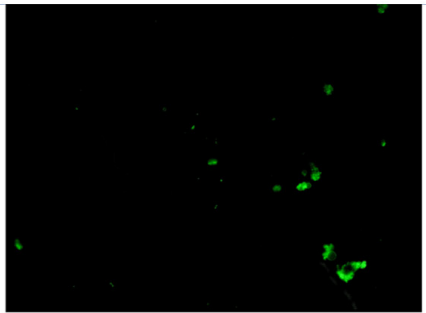

Sham

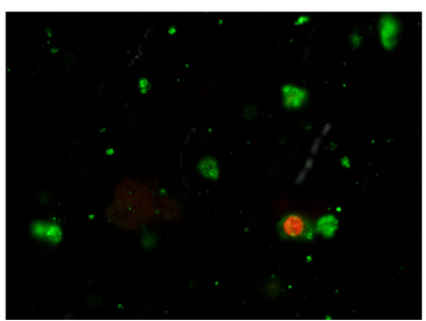

CLD

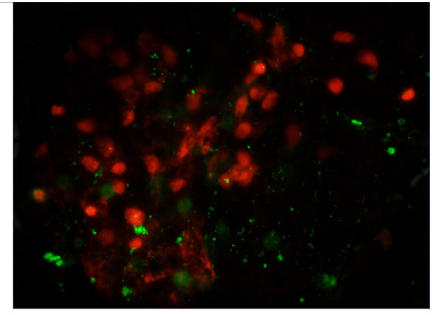

CLP

Figure 4. Inverted fluorescence microscopy analysis of apoptosis in renal cells. Annexin V-FITC/PI fluorescence staining was performed (magnification, $\mathrm{X} 400$ ). Green fluorescence represents FITC fluorescence; the cell membrane is green in the early stage of apoptosis. Red fluorescence represents PI fluorescence; the nucleus in the middle and late stage of apoptosis is red. CCD, CLP + clinical dose of ticagrelor; CLD, CLP + loading dose of ticagrelor; PI, propidium iodide.

To determine the association of neutrophils and their secreted factors with septic AKI, compared with those in the Normal group and the Sham group, serum IL-1 $\beta$ levels and MPO activity were detected in the renal tissue, and it was revealed that both MPO activity and IL- $1 \beta$ levels were increased in the CLP group, indicating that neutrophil infiltration in the renal tissue and imbalance of the inflammatory response mediated septic AKI, and that this mechanism was associated with the increase of CD62P concentration. As a cell adhesion receptor, CD62P mediates P-selectin glycoprotein ligand-1 and glycoprotein Ib interactions between platelets and neutrophils, triggering a series of cellular responses within neutrophils (28). Notably, other studies reported that CD62P protein directly induced the expression of IL-1 $\beta$ and 
other cytokines, while IL-1 $\beta$ is an important pro-inflammatory factor that could promote the bone marrow to release neutrophils, chemotactic monocytes and multinuclear cells, and infiltrate the local inflammation $(29,30)$.

To summarize, CD62P mediates the accumulation of neutrophils in the kidney, which is the initiating factor of AKI in sepsis. The activation of platelet mass initiates disseminated intravascular coagulation, which causes microcirculation disturbance of the renal tissue, and aggravates local circulatory disorders and inflammation unbalanced response in renal tissue (31). The aforementioned factors work synergistically to induce the development of septic AKI.

It was demonstrated in the present study that $\mathrm{SCr}, \mathrm{CD} 62 \mathrm{P}$, IL-1 $\beta$ and renal MPO were decreased after ticagrelor intervention in the CCD and CLD groups, and $H \& E$ staining of renal tubular epithelial cells and renal tubules was mild with no tubular deposition observed. In addition, the decrease in CD62P via ticagrelor concentration was associated with similar reductions in the renal injury marker $\mathrm{SCr}$, the proinflammatory factor IL- $1 \beta$ and MPO, the aggregation marker of central granulocytes in the kidney, suggesting that ticagrelor attenuated the severity of septic AKI by inhibiting the secretion of CD62P and the inflammatory response mediated by $\mathrm{CD} 62 \mathrm{P}$, such as reducing the release of proinflammatory factor IL-1 $\beta$, inflammatory response and chemotaxis of neutrophils, thereby reducing neutrophil accumulation in the kidney. At the same time, the expression of CD62P in the CCD group was significantly higher than the CLD group. It is speculated that there is a dose-dependent association between the inhibition of CD62P expression and ticagrelor. Certain scholars hypothesize that ticagrelor may become one of the novel targeted drugs for the treatment of septic AKI (32).

In the current study, it was demonstrated that the apoptosis rate of renal cells in CLP group was increased compared with the normal and sham groups, suggesting that renal cell apoptosis is one of the pathophysiological mechanisms underlying septic AKI. Mariano et al (33) used the plasma from patients with severe sepsis or septic shock to culture renal TECs and found that TECs still underwent functional changes and apoptosis even without hypoperfusion or hypoxia; therefore, ischemia and hypoxia may not be the primary factors of renal cell apoptosis. In the present study, it was revealed that the apoptosis rate was decreased in the CCD and CLD groups compared with the CLP group, suggesting that the inhibition of CD62P secretion using ticagrelor reduced the apoptosis rate of renal cells. The lack of caspase-3 level measurements and TUNEL staining of tissue slices are potential limitations of the current study.

In summary, the present study demonstrated that sepsis results in excessive platelet activation, causing an increase in the blood CD62P protein concentration and the renal cell apoptosis rate, which may be one of the key pathophysiological mechanisms underlying septic AKI. In sepsis, the abnormal increase in CD62P protein induces the release of the pro-inflammatory factor IL-1 $\beta$, which adheres to neutrophils, indicating that CD62P-mediated inflammation and renal cell apoptosis are the key mechanisms underlying the development of septic AKI (34). Ticagrelor exerts certain renal protective effects by inhibiting the inflammatory response mediated by
CD62P, alleviating systemic and renal local inflammation and reducing renal cell apoptosis. These findings may provide novel evidence-based insight and experimental information to aid in the prevention and treatment of septic AKI. In the current study, only animal experiments were conducted; in future, the mechanism underlying ticagrelor regulation of CD62P expression (potentially via the NF- $\mathrm{KB}$ pathway) should be examined at the cellular level and alleviating the sepsis-induced AKI.

\section{Acknowledgements}

The authors would like to thank Dr Yan-Long Pan (Department of Research and Test Center, Gansu University of Traditional Chinese Medicine, Lanzhou, China) for his help in the guidance of the experimental methods and Professor Shun Xing Zhang (Foreign Language Teaching and Research Section, Second Military Medical University, Shanghai, China), who helped revise the English in the paper.

\section{Funding}

The present work was supported by the First Hospital of Lanzhou University (grant no. ldyyyn2018-49) from the fund program named Fund of First Hospital of Lanzhou University.

\section{Availability of data and materials}

The datasets used and/or analyzed during the current study are available from the corresponding author on reasonable request.

\section{Authors' contributions}

CY made substantial contributions to conception and design, performed the experiments and participated in drafting the manuscript. CMG has confirmed the authenticity of all the raw data, and revising the manuscript critically for important intellectual content. CMG has been involved in analyzing the data and drafting the manuscript. YQM made substantial contributions to design the experiment and critically revised the manuscript for important intellectual content. NX and $\mathrm{XQW}$ contributed to acquisition and analysis of data, YQM critically revised the manuscript for important intellectual content. All authors have read and approved the final version of this manuscript.

\section{Ethics approval and consent to participate}

The present study was approved by the Research Ethics Committee of the First Hospital of Lanzhou University (Lanzhou, China).

\section{Patient consent for publication}

Not applicable.

\section{Competing interests}

The authors declare that they have no competing interests. 


\section{References}

1. Thakar CV, Christianson A, Freyberg R, Almenoff P and Render ML: Incidence and outcomes of acute kidney injury in intensive care units: A Veterans administration study. Crit Care Med 37: 2552-2558, 2009.

2. Kim WY, Huh JW, Lim CM, Koh Y and Hong SB: A comparison of acute kidney injury classifications in patients with severe sepsis and septic shock. Am J Med Sci 344: 350-356, 2012.

3. Peng Q, Zhang L, Ai Y and Zhang L: Epidemiology of acute kidney injury in intensive care septic patients based on the KDIGO guidelines. Chin Med J (Eng) 127: 1820-1826, 2014.

4. Chen YJ, Gong CL and Tan F: Effects of dexmedetomidine pretreatment on inflammatory factors and oxidative stress in rats with sepsis-induced renal injury. J Southern Med Univ 35: $1472-1475,2015$

5. Engelmann B and Massberg S: Thrombosis as an intravascular effector of innate immunity. Nat Rev Immunol 13: 34-45, 2013.

6. Schrier RW and Wang W: Acute renal failure and sepsis. N Engl J Med 35: 159-169, 2004

7. Ergin B, Kapucu A, Demirci-Tansel C and Ince C: The renal microcirculation in sepsis. Nephrol Dial Transplantat 30 169-177, 2015.

8. Evans RG, Ince C, Joles JA, Smith DW, May CN, O'Connor PM and Gardiner BS: Haemodynamic influences on kidney oxygenation: Clinical implications of integrative physiology. Clin Exp Pharmacol Physiol 40: 106-122, 2013.

9. Levy MM, Fink MP, Marshall JC, Abraham E, Angus D, Cook D, Cohen J, Opal SM, Vincent JL and Ramsay G; SCCM/ ESICM/ACCP/ATS/SIS: 2001 SCCM/ESICM/ACCP/ATS/SIS international sepsis definitions conference. Crit Care Med 31: 250-1256, 2003.

10. Yu LP: Mesh Meta-analysis of the differences in the effects of different ADP receptor antagonists on platelet function in patients with coronary heart disease. Practical Drugs Clini, 2016.

11. Thomas MR and Storey RF: The role of platelets in inflammation. Thromb Haemost 114: 449-458, 2015.

12. Woollard KJ and Chin-Dusting J: P-selectin antagonism in inflammatory disease. Curr Pharm Des 16: 4113-4118, 2010.

13. Wang Y and Chai YF: Relationship of sepsis severity and platelet change. Pract Clin Med (Jiangxi) 19: 16-18, 2018.

14. Gu X, Fu X, Wang Y, Zhang W, Fan W, Jiang Y, Hao G, Miao Q, Li Y and Zhi W: Comparison of ticagrelor and high-dose clopidogrel on the platelet functions in patients with inadequate response to clopidogrel. Am J Cardiovasc Dis 7: 1-8, 2017.

15. Zhao W and Sun GZ: Conversion of dosage between different kinds of experimental animals. Animal Husbandry Veterinary Sci Techn Infor 5: 52-53, 2012.

16. Jin H, Zhang Y, Ding Q, Wang SS, Rastogi P, Dai DF, Lu D, Purvis M, Cao C, Wang A, et al: Epithelial innate immunity mediates tubular cell senescence after kidney injury. JCI Insight 4: e125490, 2019.

17. Singbartl K and Ley K: Leukocyte recruitment and acute renal failure. J Mol Med (Berl) 82: 91-101, 2004.

18. Gu G, Gao F, Zhi Y and Yin ZX: Comparison of methods of isolation, preparation and culture about rat kidney cells. Wei Sheng Yan Jiu 34: 574-576, 2005 (In Chinese).

19. Wasung ME, Chawla LS and Madero M: Biomarkers of renal function, which and when? Clin Chim Acta 438: 350-357, 2015.
20. Yip HK, Chen SS, Liu JS, Chang HW, Kao YF, Lan MY, Chang YY, Lai SL, Chen WH and Chen MC: Serial changes in platelet activation in patients after ischemic stroke: Role of pharmacodynamic modulation. Stroke 35: 1683-1687, 2004.

21. Mullane KM, Kraemer R and Smith B: Myeloperoxidase activity as a quantitative assessment of neutrophil infiltration into ischemie myocardium. J Pharmacol Methods 14: 157-167, 1985.

22. Meduri GU, Headley S, Kohler G, Stentz F, Tolley E, Umberger R and Leeper K: Persistent elevation of inflammatory cytokines predicts a poor outcome in ARDS: Plasma IL-1beta and IL-6 levels are consistent and efficient predictors of outcome over time. Chest 107: 1062-1073, 1995.

23. Husted S and Van Giezen JJ: Ticagrelor: The first reversibly binding oral P2Y12 receptor antagonist. Cardiovas Ther 27: 259-274, 2009.

24. Cattaneo M, Schulz R and Nylander S: Adenosine-mediated effects of ticagrelor: Evidence and potential clinical relevance. J Am Coll Cardiol 63: 2503-2509, 2014.

25. Fani F, Regolisti G, Delsante M, Cantaluppi V, Castellano G, Gesualdo L, Villa G and Fiaccadori E: Recent advances in the pathogenetic mechanisms of sepsis-associated acute kidney injury. J Nephrol 31: 351-359, 2018.

26. Furie B, Furie BC and Flaumenhaft R: A journey with platelet P-selectin: The molecular basis of granule secretion, signalling and cell adhesion. Thromb Haemost 86: 214-221, 2001.

27. Lerolle N, Nochy D, Guérot E, Bruneval P, Fagon JY, Diehl JL and Hill G: Histopathology of septic shock induced acute kidney injury: Apoptosis and leukocytic infiltration. Intensive Care Med 36: 471-478, 2010.

28. Chappell D, Heindl B, Jacob M, Annecke T, Chen C, Rehm M, Conzen P and Becker BF: Sevoflurane reduces leukocyte and platelet adhesion after ischemia-reperfusion by protecting the endothelial glycocalyx. Anesthesiologists 115: 483-491, 2011.

29. Jansen MP, Florquin S and Roelofs JJ: The role of platelets in acute kidney injury. Nat Rev Nephrol 14: 457-471, 2018.

30. Lukens JR, Gurung P, Vogel P, Johnson GR, Carter RA, McGoldrick DJ, Bandi SR, Calabrese CR, Vande Walle L, Lamkanfi M and Kanneganti TD: Dietary modulation of the microbiome affects autoinflammatory disease. Nature 516: 246-249, 2014.

31. Wang TY and Zhang ZX: Progress in treatment of diffuse intravascular coagulation. Chin Pediatric Emergency Med 15: 405-407, 2008

32. Sexton TR, Zhang G, Macaulay TE, Callahan LA, Charnigo R, Vsevolozhskaya OA, Li Z and Smyth S: Ticagrelor reduces thromboinflammatory markers in patients with pneumonia. JACC Basic Transl Sci 3: 435-449, 2018

33. Mariano F, Cantaluppi V, Stella M, Romanazzi GM, Assenzio B, Cairo M, Biancone L, Triolo G, Ranieri VM and Camussi G: Circulating plasma factors induce tubular and glomerular alterations in septic burns patients. Crit Care 12: R42, 2008.

34. Li X, Li Y, Shen K, Li H and Bai J: The protective effect of ticagrelor on renal function in a mouse model of sepsis-induced acute kidney injury. Platelets 30: 199-205, 2019.

(i) $\Theta$ This work is licensed under a Creative Commons Attribution-NonCommercial-NoDerivatives 4.0 International (CC BY-NC-ND 4.0) License. 